\title{
Light bullets in an Bragg environment with carbon nanotubes in the external magnetic field
}

\author{
Yulia Nevzorova ${ }^{1}$, Ilya Dvuzhilov ${ }^{1, *}$, Sergey Boroznin ${ }^{1}$, Irina Zaporotskova ${ }^{1}$, and \\ Mikhail Belonenko ${ }^{1,2}$ \\ ${ }^{1}$ Volgograd State University, 100 University Prospect, Volgograd 400062, Russia \\ ${ }^{2}$ Volgograd Institute of Business, 63 Kachintsev Str., Volgograd 400010, Russia
}

\begin{abstract}
On the basis of the solution of the collisionless Boltzmann equation for electrons in the conduction band of semiconductor carbon nanotubes, an effective equation for the dynamics of two-dimensional few cycle light pulses (light bullets) is obtained, in the case when the environment into which carbon nanotubes are placed has a spatially modulated refractive index. The distortion of the pulse shape in the presence of a magnetic field is obtained.
\end{abstract}

\section{Introduction}

Earlier, a number of papers [1,2] studied the propagation of one-, two- and threedimensional ultrashort optical pulses in a Bragg medium with carbon nanotubes and revealed the fact of their steady propagation. It was also found that in the case where the constant magnetic field is parallel to the axis of the carbon nanotube, the shape of the pulse can change and it becomes possible to control. As shown in [3] in this case, if the same electron-radio spectrum can be found exactly and in an arbitrarily strong magnetic field, which makes it possible to analyse the differences due to the presence and absence of a constant magnetic field.

\section{Basic equations}

As a rule, the study of carbon nanotubes' electronic structure is carried out in framework of analysis of dynamics of $\pi$-electrons in strong-coupling approximation. Consider an alternating electric field that is parallel to a constant magnetic field propagating in the CNT system. The Hamiltonian for electron system can be written in the form:

$$
H=\sum_{p s} \varepsilon_{s}\left(p-\frac{e}{c} A(t)\right) a_{p s}^{+} a_{p s}
$$

"Corresponding author: dvuzhilov.ilya@,volsu.ru 
where $a_{p s}^{+}, a_{p s}$ are creation and annihilation operators of electrons at site $p$ with spin $s$, is a hopping integral, determined by the electron wave function overlapping at adjacent sites. $A(t)$ - is the light pulse electric field, $\varepsilon_{s}(p)$ - electron dispersion law.

For carbon nanotubes of the zig-zag type, the dispersion law for electrons in the presence of a magnetic field parallel to the nanotube axis is:

$$
\varepsilon_{s}\left(k_{x}, k_{y}, H\right)= \pm \gamma \sqrt{1+4 \cos \left(a p_{z} \frac{3 a k_{z}}{2}\right) \cos \left(\frac{\sqrt{3} a k_{x}}{2}\right)+4 \cos ^{2}\left(\frac{\sqrt{3} a k_{x}}{2}\right)}
$$

where $\gamma \approx 2,7 \mathrm{eV}$, ka belongs to the interval $[-\pi ; \pi], \mathrm{k}$ is the wave vector along the CNT axis, $\mathrm{a}=3 \mathrm{~b} / 2 \mathrm{~h}, \mathrm{~b}=0.152 \mathrm{~nm}$ are the distances between adjacent carbon atoms, $k_{z}$ - wave vector along the nanotube axis.

$k_{x}=\frac{2 \pi}{\sqrt{3} a M_{0}}\left(s+\frac{\Phi}{\Phi_{0}}\right) \Phi-$ magnetic flux through the cross section of the nanotube, $\Phi_{0}=\frac{\hbar c}{e}, s=1,2, \ldots, M_{0}, M_{0}-$ the number of hexagons along the perimeter of the nanotube.

Using the standard Coulomb gauge, the pulse electromagnetic field can be described by the Maxwell equations as follows:

$$
\begin{gathered}
\frac{\partial^{2} A_{z}}{\partial x^{2}}+\frac{\partial^{2} A_{z}}{\partial y^{2}}-\frac{n^{2}(x, y)}{c^{2}} \frac{\partial^{2} A_{z}}{\partial t^{2}}+\frac{q}{\pi \hbar} \sum_{m} c_{m} \sin \left(\frac{m a q}{c} A_{z}(t)\right)=0 \\
c_{m}=\sum_{m} a_{m s} b_{m s} b_{m s}=\int_{-q_{0}}^{q_{0}} d p_{z} \cos \left(\operatorname{map}_{z}\right) F_{0}(\mathbf{p})
\end{gathered}
$$

where $t$ is time, $c$ is speed of light in the medium, $n(x ; y)$ is the coefficient determining spatial variation of the carbon nanotube density, $F_{0}(\boldsymbol{p})$ - equilibrium Fermi distribution function.

Solving the problem, we neglect the diffraction spreading of the laser beam in direction along the CNT axis. We ignore the substrate electric field as well. Note that since both the typical size of CNTs and the distance between them are much smaller than the spatial domain typical size in which a few-cycle pulse is localized, we can use the continuous medium approximation and consider the current distributed over the volume.

It is established that a stable propagation of two-dimensional light bullets is possible in the presence of an external constant magnetic field directed along the nanotube axis in a Bragg medium with carbon nanotubes. Under the influence of a magnetic field, the shape of the pulse is distorted, the increase of the external magnetic field to a significant change in the propagation dynamics of a two-dimensional ultimately short optical pulse.

\section{References}

[1] M.B. Belonenko, Yu.V. Nevzorova, Bull. RAS: Physics 78, 1619 (2014)

[2] A.V. Zhukov, R. Bouffanais, M.B. Belonenko, N.N. Konobeeva, Yu.V. Nevzorova, T.F. George, Eur. Phys. J. D 69, 129 (2015)

[3] M.B. Belonenko, Yu.V. Nevzorova, E.N. Galkina, Bull. RAS: Physics 80, 837 (2016) 